\title{
Wnt Proteins
}

\author{
Karl Willert ${ }^{1}$ and Roel Nusse ${ }^{2}$ \\ ${ }^{1}$ Department of Cellular and Molecular Medicine, University of California, San Diego, California 92093 \\ ${ }^{2}$ Department of Developmental Biology, Howard Hughes Medical Institute, Stanford University School \\ of Medicine, Stanford, California 94305 \\ Correspondence: kwillert@ucsd.edu
}

Wnt proteins comprise a major family of signaling molecules that orchestrate and influence a myriad of cell biological and developmental processes. Although our understanding of the role of Wnt signaling in regulating development and affecting disease, such as cancer, has been ever increasing, the study of the Wnt proteins themselves has been painstaking and slow moving. Despite advances in the biochemical characterization of Wnt proteins, many mysteries remain unsolved. In contrast to other developmental signaling molecules, such as fibroblast growth factors (FGF), transforming growth factors (TGF $\beta$ ), and Sonic hedgehog (Shh), Wnt proteins have not conformed to many standard methods of protein production, such as bacterial overexpression, and analysis, such as ligand-receptor binding assays. The reasons for their recalcitrant nature are likely a consequence of the complex set of posttranslational modifications involving several highly specialized and poorly characterized processing enzymes. With the recent description of the first Wnt protein structure, the time is ripe to uncover and possibly resolve many of the remaining issues surrounding Wnt proteins and their interactions. Here we describe the process of maturation of Wnt from its initial translation to its eventual release from a cell and interactions in the extracellular environment.

Since the isolation of the first Wnt gene in 1982, then called int-1 (Nusse and Varmus 1982), the study of Wnt has impacted virtually all aspects of developmental biology, from establishing the polarity of a single cell within a tissue to specifying the entire body axis of an organism. At the cellular level, Wnts have been described to regulate stem cell self-renewal, apoptosis, and cell motility. During development, the requirement for Wnt can be observed at stages as early as the first cleavages of the zygote all the way to adulthood, where Wnts regulate tissue homeostasis, such as of the skin and intestine, and, when dysregulated, lead to mul- tiple disorders, such as cancer and premature aging.

Even in light of the vast literature on Wnt, currently totaling approximately 2000 PubMed citations per year, our understanding of the gene products orchestrating these diverse biological processes - the Wnt proteins-is still quite rudimentary. Early analysis of overexpressed Wnt genes, in particular Wnt1 (renamed from int-1) (Nusse et al. 1991), revealed important properties of Wnts, including their secretion from cells, glycosylation, and tight association with the cell surface and extracellular matrix. Purification of the first Wnt protein

Editors: Roel Nusse, Xi He, and Renee van Amerongen

Additional Perspectives on Wnt Signaling available at www.cshperspectives.org

Copyright (C) 2012 Cold Spring Harbor Laboratory Press; all rights reserved; doi: 10.1101/cshperspect.a007864

Cite this article as Cold Spring Harb Perspect Biol 2012;4:a007864 
(Willert et al. 2003) led to the identification of covalent protein acylation and provided insight into the poor solubility of these secreted glycosylated lipid-modified signaling molecules. However, many mysteries still surround Wnt protein biochemistry and consequently their mode of action. For example, very little is known regarding Wnt binding affinities and specificities to its many substrates, including other secreted molecules, cell surface receptors, and proteoglycans. The recent description of a highresolution crystal structure of a Wnt protein in complex with a Frizzled receptor binding domain will surely provide many important insights into how these potent growth factors engage their cognate receptors.

Here we describe the current state of our understanding of Wnt proteins from their initial translation, transit through the secretory pathway, where Wnts are extensively modified and processed, to their secretion from the cell and subsequent interactions with components of the extracellular environment. We also highlight some of the major tools available to manipulate $\mathrm{Wnt}$ proteins and interrogate their signaling pathways.

\section{THE BASICS: WNT GENES AND PREDICTED PROTEIN PRODUCTS}

All metazoan species express Wnt genes, with the genome of Hydra vulgaris carrying 13 and mice and humans carrying 19 independent genes (additional information can be found on the Wnt homepage: http://wnt.stanford.edu). Based on their primary amino acid sequence, all Wnt genes are predicted to encode secreted proteins. The defining property of Wnt proteins is a nearly invariant positioning of 22 cysteine residues, most of which are postulated to form disulfide bridges that maintain a globular secondary structure. Some studies have suggested that Wnt proteins exist as dimers (Burrus and McMahon 1995; Cha et al. 2008) maintained by intermolecular disulfide linkages; however, this has not been observed to be the case for purified and biologically active $W n t$ proteins. In addition, the recent high-resolution structure of a Wnt protein suggests that all conserved cysteine residues are occupied in intramolecular rather than intermolecular disulfide bridges (Janda et al. 2012). Wnt proteins carry several stretches of highly charged amino acids and have a predicted isoelectric point of nearly 9 , which, in combination with multiple glycosylation events, would lead one to expect that Wnt proteins are readily soluble in an aqueous environment.

The primary amino acid sequence of Wnt shows several hallmarks of secreted proteins, most notably a signal sequence for secretion, a stretch of approximately 20 hydrophobic amino acids. Cleavage of this signal peptide can be predicted using several computer algorithms; however, the true first amino acid of a Wnt protein was identified by amino-terminal sequencing of a purified Wnt protein (Willert et al. 2003). Interestingly, in the case of Wnt5a, the aminoterminal residue was found to be located 62 amino acids from the predicted translational start site (Mikels and Nusse 2006). This observation serves as a cautionary note for those who wish to append amino-terminal tags (His, HA, or GFP) onto Wnts, because such tags may be cleaved from the mature protein upon signal sequence cleavage. In addition, with only a few exceptions, tagged Wnt genes produce proteins with significantly lower activity (K Willert and R Nusse, unpubl.), and caution should be exercised with the interpretation of any data using tagged Wnt genes.

Aside from targeting Wnt proteins for secretion, the amino terminus may harbor additional critical biological functions. A survey of isoform and alternative splicing databases reveals that multiple Wnt genes carry distinct $5^{\prime}$ untranslated regions (UTRs) and are predicted to encode distinct amino termini. In the case of Wnt16, two isoforms with distinct $5^{\prime}$ UTRs are expressed from alternative promoters (Fear et al. 2000). Although little is known regarding the biological significance of these two isoforms, it is intriguing that one of the two isoforms has a broad expression pattern, whereas the other is restricted to the pancreas. Changes in the amino terminus of Wnt proteins may represent a common mechanism by which signaling activity can be affected, as indicated by the studies on Tiki, a gene encoding a transmembrane 
protein that antagonizes Wnt signaling (Zhang et al. 2012). Tiki protein acts cell-autonomously as a protease to cleave eight amino terminal residues from a Wnt protein, thereby reducing receptor-binding and signaling activities. Such observations suggest that the Wnt family of proteins is significantly more complex and diverse than expected for 19 genes.

It should be noted that in contrast to Wnt signaling, which has been conveniently-and perhaps inappropriately_categorized as either canonical or noncanonical, no sequence or structural basis for this distinction has been identified in Wnt proteins. Although many studies make reference to either canonical or noncanonical Wnts, this difference is most likely conferred by cellular context as determined by the expressed repertoire of receptors and signal transducers rather than by an intrinsic property of the Wnt proteins. The hypothesis that Wnt signaling activity is conferred by cellular context rather than by the Wnt protein sequence is supported by the observations that a so-called noncanonical Wnt5a can act "canonically" by activating $\beta$-catenin signaling in certain contexts (He et al. 1997; Mikels and Nusse 2006). Furthermore, maternal Wnt11, which has been largely studied for its roles in noncanonical Wnt signaling, specifies the dorsal axis in Xenopus by localizing $\beta$-catenin to dorsal nuclei, thus promoting a canonical signaling pathway (Tao et al. 2005).

\section{INTRACELLULAR PROCESSING OF WNT}

Upon translation and targeting to the endoplasmic reticulum (ER), Wnt proteins associate with multiple processing enzymes that chaperone Wnts on their journey to the extracellular space. The intermediate vesicle compartments through which Wnt passes and the order in which these posttranslational modifications are added have not been precisely elucidated. The two most prominent modifications on Wnt are glycosylation and acylation. The number of glycosylation attachments varies significantly between Wnts, for example, Wnt 1 carries four and Wnt3a carries two $\mathrm{N}$-linked glycosylations, whereas the most distantly related Wnt homolog, Drosophila WntD, appears to be devoid of any glycosylations or any modification because its mass is identical to the predicted mass based on amino acid content (Ching et al. 2008). When overexpressed, Wnts often resolve as multiple bands in immunoblots, which return to a single species upon treatment with glycosidases or site-directed mutagenesis of the individual predicted glycosylation sites. Interestingly, in the rare cases in which Wnt proteins have been detected in soluble form (Wnt1, Wnt3a, Wnt5a, Wingless [Wg], and WntD), the protein migrates as a single species, suggesting that upon complete intracellular processing, the composition of Wnt is quite homogeneous. Site-directed mutagenesis of individual glycosylation sites has minor effects on Wntl activity in overexpression assays (Mason et al. 1992), whereas secretion of Wnt3a and Wnt5a glycosylation mutants is significantly impaired (Komekado et al. 2007; Kurayoshi et al. 2007), suggesting that glycosylation likely plays a critical role in control over Wnt folding and subsequent secretion.

In contrast to glycosylation, acylation is absolutely essential for Wnt activity. Although early studies suggested that glycosylation is not critical for Wnt signaling (Mason et al. 1992), subsequent studies have argued that nonglycosylated Wnt is not acylated and consequently not secreted (Komekado et al. 2007). Initial mass spectrometry studies of purified Wnt3a identified two types of covalently attached acyl groups: a palmitic acid linked via a thioester to a conserved cysteine residue (Willert et al. 2003) and a palmitoleic acid linked via an oxyester to a conserved serine residue (Takada et al. 2006). However, the recently published crystal structure of Xenopus Wnt8 in complex with a Frizzled CRD indicates that only the conserved serine residue is lipidated, whereas the conserved cysteine is occupied in a disulfide linkage, thus arguing that Wnt proteins are modified by a single lipid moiety (Janda et al. 2012). Mutagenesis of the serine lipid modification sites (S209 in mouse Wnt3a) yielded nonfunctional and poorly secreted protein (Takada et al. 2006). In contrast, Wg carrying a mutation of the predicted acyl-modified serine $(\mathrm{S} 239)$ is 
secreted and shows poor signaling activity (Franch-Marro et al. 2008a). Despite this apparent discrepancy between Wnt3a and Wg, the emerging model is that acylation of Wnt is required for proper intracellular processing and signaling activity.

The mechanism by which a lipid is attached to the Wnt polypeptide backbone has not been elucidated at the biochemical level. However, the critical role for the resident ER protein Porcupine (Porcn) in Wnt processing (van den Heuvel et al. 1993; Kadowaki et al. 1996) and its homology to membrane-bound $\mathrm{O}$-acyltransferases (MBOAT) (Hofmann 2000) have led to the hypothesis that Porcn catalyzes the transfer of a lipid to serine. The requirement for acylation in Wnt function is highlighted by the fact that mutations in Porcn ablate all Wnt signaling and result in early embryonic lethality in mice (Barrott et al. 2011; Biechele et al. 2011) with embryos failing to gastrulate and form mesoderm. Mutations in human PORCN, an $\mathrm{X}$-linked gene, lead to a rare genetic disorder, called focal dermal hypoplasia (FDH), characterized by skin abnormalities and a host of developmental malformations and defects (Grzeschik et al. 2007; Wang et al. 2007). Mutations in $P O R C N$ are lethal in males, consistent with the early embryonic lethality observed in mouse knockouts. In contrast, females survive, albeit with a host of defects, owing to random X-inactivation, which produces mosaic PORCN expression with cells that express PORCN rescuing those that do not.

Although glycosylation and acylation appear to occur on all Wnt proteins (with the exception of the distantly related Drosophila WntD) (Ching et al. 2008), several other posttranslational modifications, which were not revealed in the high-resolution structure of Xenopus Wnt8, may be restricted to specific Wnts. For example, tyrosine sulfation has been shown to regulate Wnt5a and 11 hetero-oligomer formation, and the resulting Wnt multimer shows higher signaling activity than either individual Wnt (Cha et al. 2009). Using antisense oligonucleotides to deplete specific maternal mRNAs, the investigators of this study showed that the enzyme tyrosyl-protein sulfotransferase-1
(TPST-1) is required for Xenopus dorsal axis formation and for $O$-sulfation of specific tyrosine residues on Wnt5a and 11.

Additionally, the ER-resident Oto protein may promote the addition of glycophosphatidylinositol (gpi)-like anchors to Wnt1 and 3a, thereby increasing their hydrophobicity and ER retention (Zoltewicz et al. 2009), although the site of this modification is not known. Overexpression of gpi-specific phospholipase D (GPIPLD) leads to the release of Wnt protein, presumably by cleavage of the gpi anchor. Interestingly, the phospholipases D1 and D2 are direct targets of Wnt/ $\beta$-catenin signaling and act in a positive-feedback loop to increase signaling in cancer (Kang and Min do 2010; Kang et al. 2010). A possible mechanism for this increase in Wnt signaling may be through the release of ER-retained Wnt proteins. These studies also provide a possible mechanistic basis for poor Wnt secretion.

\section{STRUCTURE OF WNT PROTEINS}

While it required 20 years to obtain a pure and biologically active Wnt protein, it took another 10 years to achieve its crystallization and provide a high-resolution structure of a Wnt protein. The feat was accomplished in Chris Garcia's laboratory and has revealed several interesting properties of Wnt proteins (Janda et al. 2012). The $3.25 \AA$ crystal structure was determined for Xenopus Wnt8 (XWnt8) in complex with the CRD of mouse Frizzled8 and reveals a highly unusual two-domain structure with aminoterminal and carboxy-terminal domains (NTD and CTD) forming a protein fold previously not identified in any other protein structure (Fig. 1). The NTD is composed of a cluster of $\alpha$-helices with 10 of the conserved cysteine residues forming five disulfide bridges, whereas the CTD is dominated by two $\beta$-sheets and maintained by six disulfide bridges.

In this structure, Wnt extends a thumb from the NTD and an index finger from the CTD to grasp the globular Frizzled CRD. Interestingly, the thumb extends a lipid at serine 187, the highly conserved residue previously identified to carry a covalently attached palmitoleic acid 
A

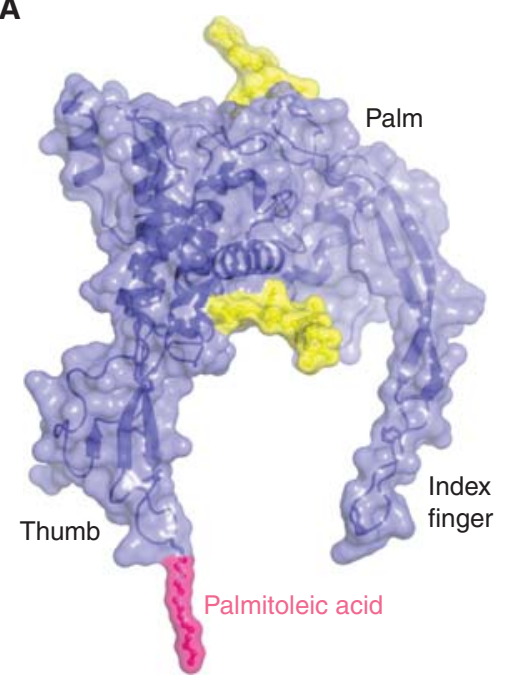

B

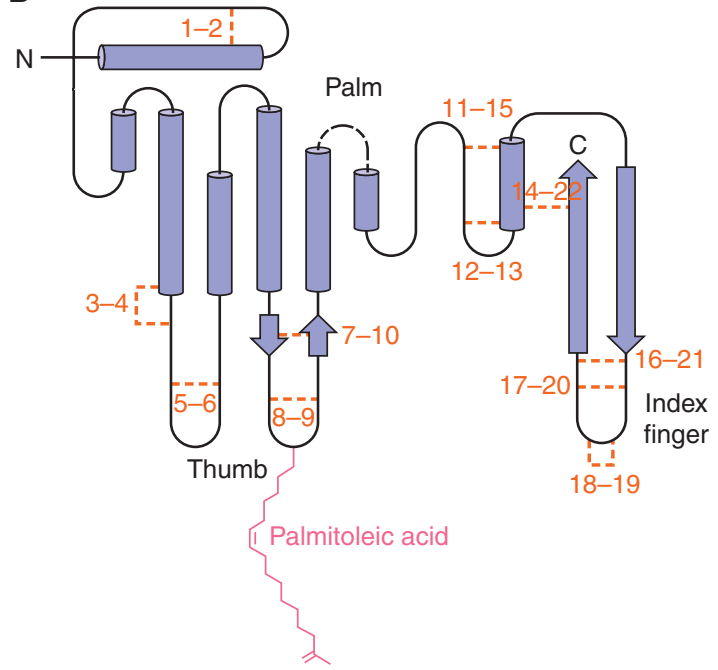

Figure 1. Structure of Wnt. (A) Space-filling model of XWnt8. The Frizzled CRD structure has been removed. (Yellow clusters) The $N$-linked glycosylations in XWnt8. (B) Secondary structure for Wnt. (Orange) The conserved 22 cysteine residues are numbered to indicate the pairs that form disulfide bridges. $N$-linked glycosylations are not shown because the numbers and positions of $N$-linked glycosylations are highly variable among Wnts. (Dashed line) The approximate position of the linker region where $\mathrm{Wg}$ carries an insert of about 80 amino acids. (Figures were generated with the kind assistance of C. Janda and C. Garcia, Stanford University.)

(Takada et al. 2006). This protein-lipid thumb structure is nestled in a deep hydrophobic groove of the CRD, where it makes multiple contacts to completely traverse the CRD surface. As is the case with the thumb, the points of contact of the index finger in the CTD with the CRD are also dominated by hydrophobic and highly conserved residues. Some of the contact points on the Frizzled8 CRD are substituted in other Frizzled CRDs, thus providing a possible mechanism to influence Wnt-Frizzled binding specificities. To further extend the hand analogy for the Wnt structure, the region between the thumb and the index finger is akin to the palm, where the NTD and CTD are intimately associated. Interestingly, the solvent-exposed linker region between NTD and CTD corresponds to the region with the greatest flexibility among Wnt proteins; in particular, Wg carries a large insert of 80 amino acids not present in other Wnts. This "Wg insert" has been used to generate arguably the best antisera to any Wnt protein (van den Heuvel et al. 1989,
1993), further supporting the model that this nonconserved linker region is solvent exposed and likely not involved in binding to the Frizzled CRD.

This Wnt structure will enable a more rational approach to interrogate Wnt-Frizzled interactions and design Wnt agonists and antagonists. A remaining unanswered question concerns the structure of an uncomplexed Wnt protein. The covalently attached lipid necessitates some type of interaction, either with a carrier protein, such as Swim (Mulligan et al. 2012), or with membranous domains, to shield this hydrophobic moiety in a largely aqueous environment. The highly accessible presentation of the lipid moiety on Wnt makes the interaction with carrier molecules or the plasma membrane a likely scenario. An alternative possibility is that the hydrophobic portions of the thumb and index finger fold in to form a fist; however, the current crystal structure does not provide evidence for such intramolecular folding. 
K. Willert and R. Nusse

\section{SECRETION OF WNT}

As ER and Golgi-processed Wnt protein is targeted for secretion (Fig. 2), a sorting receptor encoded by the Wntless gene (Wls, also known as Eveness interrupted/Evi, Sprinter, MIG-14, and Gpr177), a multispan transmembrane protein, binds and accompanies Wnt to the cell surface (Banziger et al. 2006; Bartscherer et al. 2006; Goodman et al. 2006). Wls binding of Wnt3a requires acylation on Ser-209, indicating that it acts downstream from Porcn. A comprehensive mutational analysis indicated that acylation of the Ser equivalent of $\mathrm{Wg}^{\mathrm{S} 239}$ is required for the interaction of Wls with all Wnts, except WntD (Herr and Basler 2011). Furthermore, by using a membrane-tethered Wg pro- tein, WgNRT (Zecca et al. 1996), Herr and Basler provided evidence that mere membrane association is not sufficient for $\mathrm{Wg}$ association with Wls, thus suggesting that acylation by Porcn enables Wnt's functional interaction with Wls. In addition, in the case of Wg, glycosylation on conserved residues does not affect the dependence of Wnt on Wls.

Vacuolar acidification is required for release of Wnt protein (Coombs et al. 2010), and small drug inhibition of the V-ATPase, a proton pump required for vacuolar acidification, prevents Wls from releasing Wnt so that Wnt-Wls complexes accumulate both in cells and at the plasma membrane (Coombs et al. 2010). However, although essential, a decrease in $\mathrm{pH}$ is not sufficient to dissociate the Wnt-Wls complex.

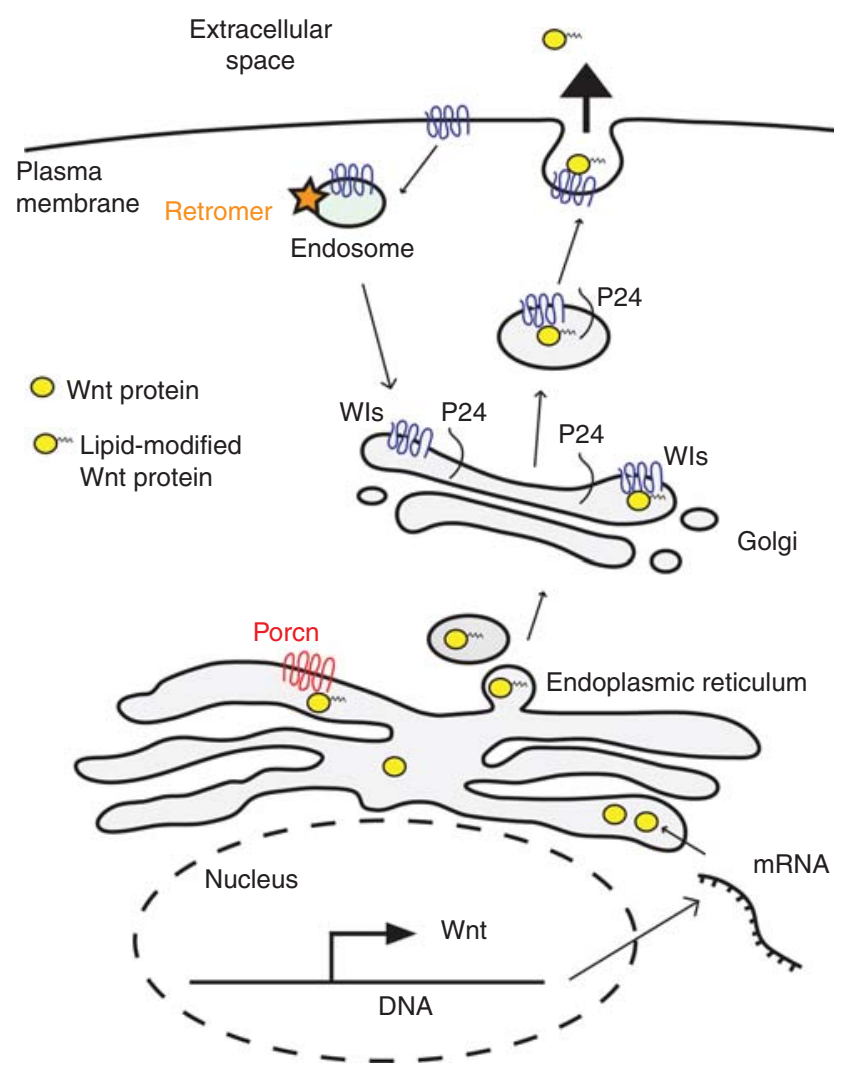

Figure 2. Wnt secretion. Upon translation, Wnt proteins (yellow ovals) undergo a series of modifications as they transit through the secretory pathway and associate with several proteins, including Porcn in the endoplasmic reticulum and Wls in the Golgi apparatus. Efficient secretion of Wnt also requires the recycling of Wls via the retromer complex. 
RNA interference screens in two separate laboratories identified members of the $\mathrm{p} 24$ protein family as cargo receptors for Wnt in anterograde transport and secretion (Buechling et al. 2011; Port et al. 2011). Although the two groups differ slightly on which of the nine fly p24 family members are involved in Wg secretion, they agree in the basic finding that these cargo proteins specifically regulate $\mathrm{Wg}$ secretion and that secretion of other signaling molecules (e.g., Decapentaplegic, Hedgehog, and Unpaired) is unaffected. Therefore, Wnt protein does not exit a cell through passive transport or bulk flow but requires specific cargo proteins, such as the p24 family of highly conserved transmembrane receptors, to exit from the ER.

Once Wnt is released from the cell, Wls is recycled via endosomes and the retromer complex to the Golgi, where it acts again to escort a newly processed Wnt protein to the cell surface (Coudreuse et al. 2006; Prasad and Clark 2006; Belenkaya et al. 2008; Franch-Marro et al. 2008b; Port et al. 2008; Yang et al. 2008). As a result, interfering with either Wls expression or its recycling via the retromer inhibits Wnt secretion. In contrast to the classical retromer complex, which involves the sorting nexins SNX1SNX2 and SNX5-SNX6 (referred to as SNXBAR sorting nexins) and cargo-selective VPS26, VPS29, and VPS35, Wls recycling requires the distantly related sorting nexin SNX3 (Harterink et al. 2011; Zhang et al. 2011). Therefore, retrograde transport of Wls is distinct from other recycled cargo, raising the possibility of specifically interfering with Wnt secretion through targeted disruption of Wls recycling via SNX3.

If retromer components Vps35 and Vps26 are mutant, Wls is targeted for degradation rather than for endosomal recycling to the Golgi so that it is no longer available to facilitate Wnt secretion. Such defects in the retromer complex can be rescued by overexpression of Wls. In Caenorhabditis elegans, it has been shown that the recycling of the Wls homolog MIG-14 from the cell surface to the Golgi requires the MTM6/MTM-9 myotubularin complex, which dephosphorylates $\mathrm{PIP}_{3}$, a central regulator of endosomal trafficking (Silhankova et al. 2010). A conserved endocytosis motif required for Wls recycling has been identified in the third intracellular loop of Wls; its mutation results in Wls accumulation on the cell surface and impairs $\mathrm{Wg}$ secretion and signaling (Gasnereau et al. 2011).

Although it is clear that the Wls/retromer system is essential for Wnt secretion, studies performed in flies and worm have reached apparently contradicting conclusions with respect to the signaling range of Wnt secreted from retromer-defective cells. Long-range, but not short-range action of Egl-20 (a worm Wnt) was impaired in retromer mutants, suggesting that the retromer complex is important for packaging Wnt for long-range signaling (Coudreuse et al. 2006). In contrast, flies carrying mutations in Vps35 are defective in short-range signaling as evidenced by strong reduction in expression of the Wg target senseless (FranchMarro et al. 2008b).

In motor neurons in Drosophila, Wls not only transports Wnt from the Golgi to the plasma membrane, but also functions to shuttle Wnt across the synaptic cleft of the neuromuscular junction in exosome-like vesicles (Korkut et al. 2009). Furthermore, postsynaptically in the muscle, Wls also guides Wg-activated Frizzled-2 trafficking before Frizzled-2 is proteolytically cleaved and its carboxyl terminus is imported into the nucleus (Mathew et al. 2005; Ataman et al. 2006; Korkut et al. 2009).

Structural modeling suggests that the ER luminal portion of Wls contains a lipocalinfamily fold (Coombs et al. 2010), which has been shown to interact with lipids. Interestingly, a lipocalin fold has also been predicted by NMR analysis of the Wnt inhibitory factor (WIF) domain (Liepinsh et al. 2006; Malinauskas 2008), which is found in the secreted Wnt antagonist WIF1 and the Wnt receptor Ryk. Furthermore, a fly lipocalin, named Swim (secreted Wnt interacting molecule) has been proposed to act as Wnt chaperone and shield the hydrophobic moieties on Wnt, thereby enabling efficient Wnt diffusion or transport in a largely aqueous environment (Mulligan et al. 2012). Expression of certain lipid-binding proteins, including Lipocalin2 and FABP5, is up-regulated in cells overexpressing Wnt1 (Ziegler et al. 2005, 
2007). Additionally, Wls/Gpr177 is also a target of Wnt signaling in mouse cells (Fu et al. 2009), suggesting the presence of feed-forward regulation that ensures sufficient chaperones are available to usher Wnt through the secretory pathway. However, in flies, Wls appears not to be a target of canonical Wnt signaling (Herr and Basler 2011). Alternatively, it is tempting to speculate that Wnt expression triggers a signaling system similar to that of the unfolded protein response, thereby activating expression of genes that encode fatty acid-binding proteins, such as lipocalins, which facilitate the export of hydrophobic Wnt proteins from the ER and to the cell surface. During the transit from the Golgi to the plasma membrane, Wnt protein is passed from Wls to a lipocalin, which then accompanies it in the extracellular space and may regulate its distribution. However, it has been shown that the secretion of the Swim protein is independent of Wg (Mulligan et al. 2012).

Following release from the secretory machinery, it has been suggested that Wnts become tethered to the plasma membrane via their lipid moieties, a feasible model given the lipid's accessibility as revealed by the protein structure (Janda et al. 2012). Although this is a formal possibility, it is clear that a significant proportion of Wnt (e.g., Wnt3a and Wnt5a) can be purified in a biologically active form from conditioned media. Alternatively, Wnt proteins may become incorporated into lipoprotein complexes. In Drosophila, Wg (and $\mathrm{Hh}$ ) was found to colocalize and associate with the lipoprotein Lipophorin, a particle with similar characteristics to ApoB-based lipoproteins (Panakova et al. 2005). Knockdown of Lipophorin expression by RNA interference significantly reduced the signaling range of both $\mathrm{Wg}$ and $\mathrm{Hh}$, suggesting that lipoprotein complexes regulate long-range Wnt signaling. In mammalian cells, Wnt3a was shown to cofractionate by $\mathrm{KBr}$ isopycnic density centrifugation with the lipoprotein marker hApoB100 and was associated with both high- and low-density lipoproteins (HDL and LDL) (Neumann et al. 2009). Furthermore, addition of HDL, but not LDL, supported Wnt3a solubility in medium containing delipidated fetal calf serum. Taken together with its hydrophobic properties, Wnt solubility in the extracellular environment necessitates molecules or complexes that interact with hydrophobic moieties.

\section{WNTS IN THE EXTRACELLULAR ENVIRONMENT}

As they reach the cell surface, Wnt proteins encounter a myriad of interacting molecules. Early biochemical studies revealed a high affinity of Wnts for polyanionic compounds, including heparin and suramin (Brown et al. 1987; Papkoff et al. 1987; Bradley and Brown 1990; Burrus and McMahon 1995). Multiple biochemical and genetic studies since then have underscored the importance of these interactions between Wnt and extracellular carbohydrate chains. Multiple genes involved in heparan sulfate (HS) synthesis, including sugarless, sulfateless, tout-velu, sister of tout-velu, and brother of tout-velu, regulate $\mathrm{Wg}$ signaling (Binari et al. 1997; Hacker et al. 1997; Haerry et al. 1997; Lin and Perrimon 1999; Bornemann et al. 2004; Han et al. 2004; Takei et al. 2004). Although mutations in these genes do not completely block signaling, clear $w g$-related phenotypes are observed, suggesting an important modulatory role for these enzymes in Wnt signaling.

Biochemical studies have revealed that components of the extracellular space, such as Glypicans (Capurro et al. 2005a,b) and Biglycan (Berendsen et al. 2011), modulate Wnts-receptor interactions and can significantly influence signaling output and strength. Mutations of zebrafish Knypek, a heparan sulfate proteoglycan (HSPG), leads to defects in convergent extension movements, a process regulated by Wnt11 (Topczewski et al. 2001) and controlling the location of the centrosome/microtubule-organizing center (MTOC) relative to the cell nucleus and the body axes (Sepich et al. 2011). HSPGs have also been found to maintain solubility and activity of Wnt protein and prevent their aggregation in low serum conditions (Fuerer et al. 2010). Furthermore, the degree of $O$ sulfation of HS has been shown to regulate Wnt signaling activity (Ai et al. 2003). Desulfation of heparin and HS chains of Glypican 1 by the cell 
surface sulfatase QSulf1 reduces Wnt binding, thus producing a low-affinity HS-Wnt complex that can more effectively engage their receptors and initiate Wnt signal transduction.

In addition to their complex interactions with glycans in the extracellular space, Wnts encounter multiple protein-binding partners, including WIF, Sfrp, and cell surface receptors. Subsequent articles in this collection address these interactions in detail.

\section{TOOLS AND METHODS TO STUDY WNT PROTEINS AND SIGNALING}

\section{Wnt Proteins}

The purification of Wnt eluded many attempts for two decades. Initial purification strategies focused on the founding Wnt protein, Wnt (initially referred to as Int-1), and found it to be poorly soluble and largely associated with the extracellular matrix and the cell surface (Brown et al. 1987; Papkoff et al. 1987). The first cell-free and biologically active Wnt proteins were $\mathrm{Wg}$ (van Leeuwen et al. 1994) and mouse Wnt3a (Shibamoto et al. 1998). These soluble Wnt proteins have been used extensively in the form of conditioned medium harvested from cells overexpressing Wnt. Although convenient, these Wnt-conditioned media harbor countless contaminating proteins and activities that may complicate the interpretation of any biological assay.

Despite extremely low expression levels $(\sim 100 \mu \mathrm{g} \mathrm{Wnt3a/L})$, a sensitive and reliable activity assay involving $\beta$-catenin stabilization enabled the purification of the first biologically active Wnt protein, Wnt3a (Willert et al. 2003). This purification scheme has been applied to other Wnt proteins with varying degrees of success. Whereas Wnt3a and Wnt5a (Mikels and Nusse 2006) were readily purified using nearly identical protocols, purification of other Wnt proteins has proven more difficult because they tend to form protein aggregates lacking biological activity (K Willert and R Nusse, unpubl.). At present, the reasons for such differences in the biochemical properties of Wnts are unknown, leaving us with the current conclusion that not all Wnt proteins are created equal. Further advances in Wnt protein biochemistry are certain to come, and the recently published high-resolution structure of a Wnt protein (Janda et al. 2012) will likely provide insight into many remaining mysteries in Wnt biochemistry.

\section{Wnt Agonists and Antagonists (Proteins)}

Aside from Wnts, several other proteins with Wnt signaling activities have been identified, including Norrin (Xu et al. 2004; Junge et al. 2009) and R-spondin (Kazanskaya et al. 2004; Kim et al. 2006; Nam et al. 2006). Recent studies identified the receptor for R-spondin to be encoded by the Lgr5 homologs (Carmon et al. 2011; de Lau et al. 2011; Glinka et al. 2011). Interestingly, Lgr5, which was previously identified as a Wnt target gene and stem cell marker in intestinal crypts (Barker et al. 2007), associates with the Frizzled/Lrp Wnt-receptor complexes. Therefore, the emerging model involves a complex of cell surface molecules in which Wnt signals acting through Frizzled/Lrp are potentiated by an R-spondin/Lgr receptor complex. Removal of R-spondin1 or mutation of Lgr4/5 in cultured crypt organoids can be rescued by small molecule Wnt pathway agonists (see below), suggesting that the R-spondin/Lgr signaling axis serves to enhance Wnt signaling. Taken together, although R-spondins by themselves have no known intrinsic Wnt signaling activity, they may serve to elevate and thereby uncover endogenous Wnt signaling activities.

Several genetic and biochemical studies have identified multiple secreted proteins that potently antagonize Wnt signaling, including DKK, Sfrp, WIF (for review, see Cruciat and Niehrs 2012), and Klotho (Liu et al. 2007). The protein Wise/Sclerostin can act both as an activator or inhibitor depending on cellular context (Itasaki et al. 2003). These proteins and their mode of action are discussed in detail in a subsequent article. Additionally, several Wnt antagonists have been engineered by fusing Wnt-binding domains, such as the cysteinerich domain (CRD) of Frizzled, to an affinity tag, such as the constant region of human immunoglobulin heavy chain. 
Wnt Agonists and Antagonists (Small Molecules)

The most widely used class of small molecule agonists of Wnt signaling inhibits GSK3. Most prominent among these is lithium, which at millimolar concentrations potently inhibits GSK3 and leads to $\beta$-catenin stabilization (Klein and Melton 1996). Several other small molecule GSK3 inhibitors with $\mathrm{IC}_{50}$ values in the nanomolar to micromolar ranges have been identified (Bregman et al. 2004; Sato et al. 2004) and are commercially available. These GSK3 inhibitors potently activate the Wnt pathway, but their use can be problematic because GSK3 represents a major signaling hub for multiple other signaling pathways and its inhibition will most certainly affect other pathways in addition to Wnt.

A chemical library screen led to the identification of two potent and highly specific inhibitors of Wnt signaling, called IWP and IWR (Chen et al. 2009). IWR interacts and stabilizes Axin protein, thereby accelerating $\beta$-catenin degradation. IWP interacts with PORCN and inhibits its acyl transferase activity. Because PORCN activity is essential for processing of all Wnts, its inhibition will likely block secretion of all Wnts, thus producing the equivalent of an "all Wnt mutant phenotype." Importantly, inhibition of endogenous Wnt signaling with this drug in cell culture can be readily rescued by providing exogenous Wnt protein (e.g., see Ten Berge et al. 2011). In addition, because PORCN function is dedicated to Wnt processing, it is likely that treatment of cells with IWP will not affect any other processes besides Wnt signal transduction.

\section{CONCLUDING REMARKS}

With their diverse functions and roles in countless biological processes, isolation and further characterization of Wnt proteins will certainly provide insights into mechanisms and modes of action. Its interactions with receptors, coreceptors, proteoglycans, and secreted proteins underscore the critical role of Wnt proteins as major players in regulating and controlling cell behavior and fate. Many obstacles still need to be overcome to provide us with a better understanding of Wnts' actions. For example, measuring affinities with which each of the 19 Wnts interacts with the various components it encounters in the extracellular environment will lead to a better understanding of how signaling specificities are achieved. In addition, identification and characterization of Wnt-containing complexes (e.g., Wnt-Rspondin-Fzd-Lgr) is critical in controlling Wnt activities, because formation of such complexes likely affects signaling outputs. Finally, with the first crystal structure of a Wnt protein in hand, we will now be able to take a more rational approach in elucidating and dissecting Wnt interactions and binding activities.

\section{ACKNOWLEDGMENTS}

We are grateful to C.Y. Janda and K.C. Garcia for their kind assistance in generating the spacefilling model of Wnt shown in Figure 1. Research on Wnt proteins in our laboratories is supported by grants from the California Institute of Regenerative Medicine (RB1-010406 to K.W. and RC1-00133-1 to R.N.). K.W. has support from the National Institutes of Health and start-up funding from the UCSD Stem Cell Program. R.N. is a Howard Hughes Investigator.

\section{REFERENCES}

\footnotetext{
* Reference is also in this collection.
}

Ai X, Do AT, Lozynska O, Kusche-Gullberg M, Lindahl U, Emerson CP Jr. 2003. QSulf1 remodels the 6-O sulfation states of cell surface heparan sulfate proteoglycans to promote Wnt signaling. J Cell Biol 162: 341-351.

Ataman B, Ashley J, Gorczyca D, Gorczyca M, Mathew D, Wichmann C, Sigrist SJ, Budnik V. 2006. Nuclear trafficking of Drosophila Frizzled-2 during synapse development requires the PDZ protein dGRIP. Proc Natl Acad Sci 103: 7841-7846.

Banziger C, Soldini D, Schutt C, Zipperlen P, Hausmann G Basler K. 2006. Wntless, a conserved membrane protein dedicated to the secretion of Wnt proteins from signaling cells. Cell 125: 509-522.

Barker N, van Es JH, Kuipers J, Kujala P, van den Born M, Cozijnsen M, Haegebarth A, Korving J, Begthel H, Peters PJ, et al. 2007. Identification of stem cells in small intestine and colon by marker gene Lgr5. Nature 449: $1003-$ 1007.

Barrott JJ, Cash GM, Smith AP, Barrow JR, Murtaugh LC. 2011. Deletion of mouse Porcn blocks Wnt ligand 
secretion and reveals an ectodermal etiology of human focal dermal hypoplasia/Goltz syndrome. Proc Natl Acad Sci 108: $12752-12757$.

Bartscherer K, Pelte N, Ingelfinger D, Boutros M. 2006 Secretion of Wnt ligands requires Evi, a conserved transmembrane protein. Cell 125: 523-533.

Belenkaya TY, Wu Y, Tang X, Zhou B, Cheng L, Sharma YV, Yan D, Selva EM, Lin X. 2008. The retromer complex influences Wnt secretion by recycling Wntless from endosomes to the trans-Golgi network. Dev Cell 14: $120-131$.

Berendsen AD, Fisher LW, Kilts TM, Owens RT, Robey PG, Gutkind JS, Young MF. 2011. Modulation of canonical Wnt signaling by the extracellular matrix component biglycan. Proc Natl Acad Sci 108: 17022-17027.

Biechele S, Cox BJ, Rossant J. 2011. Porcupine homolog is required for canonical Wnt signaling and gastrulation in mouse embryos. Dev Biol 355: 275-285.

Binari RC, Staveley BE, Johnson WA, Godavarti R, Sasisekharan R, Manoukian AS. 1997. Genetic evidence that heparin-like glycosaminoglycans are involved in wingless signaling. Development 124: 2623-2632.

Bornemann DJ, Duncan JE, Staatz W, Selleck S, Warrior R. 2004. Abrogation of heparan sulfate synthesis in Drosophila disrupts the Wingless, Hedgehog and Decapentaplegic signaling pathways. Development 131: 1927-1938.

Bradley RS, Brown AM. 1990. The proto-oncogene int-1 encodes a secreted protein associated with the extracellular matrix. EMBO J 9: 1569-1575.

Bregman H, Williams DS, Atilla GE, Carroll PJ, Meggers E. 2004. An organometallic inhibitor for glycogen synthase kinase 3. J Am Chem Soc 126: 13594-13595.

Brown AM, Papkoff J, Fung YK, Shackleford GM, Varmus HE. 1987. Identification of protein products encoded by the proto-oncogene int-1. Mol Cell Biol 7: 3971-3977.

Buechling T, Chaudhary V, Spirohn K, Weiss M, Boutros M. 2011. p24 proteins are required for secretion of Wnt ligands. EMBO Rep 12: 1265-1272.

Burrus LW, McMahon AP. 1995. Biochemical analysis of murine Wnt proteins reveals both shared and distinct properties. Exp Cell Res 220: 363-373.

Capurro MI, Shi W, Sandal S, Filmus J. 2005a. Processing by convertases is not required for glypican-3-induced stimulation of hepatocellular carcinoma growth. J Biol Chem 280: 41201-41206.

Capurro MI, Xiang YY, Lobe C, Filmus J. 2005b. Glypican-3 promotes the growth of hepatocellular carcinoma by stimulating canonical Wnt signaling. Cancer Res 65: 6245-6254.

Carmon KS, Gong X, Lin Q, Thomas A, Liu Q. 2011. Rspondins function as ligands of the orphan receptors LGR4 and LGR5 to regulate Wnt/ $\beta$-catenin signaling. Proc Natl Acad Sci 108: 11452-11457.

Cha SW, Tadjuidje E, Tao Q, Wylie C, Heasman J. 2008. Wnt5a and Wnt11 interact in a maternal Dkk1-regulated fashion to activate both canonical and non-canonical signaling in Xenopus axis formation. Development 135: 3719-3729.

Cha SW, Tadjuidje E, White J, Wells J, Mayhew C, Wylie C, Heasman J. 2009. Wnt11/5a complex formation caused by tyrosine sulfation increases canonical signaling activity. Curr Biol 19: 1573-1580.

Chen B, Dodge ME, Tang W, Lu J, Ma Z, Fan CW, Wei S, Hao W, Kilgore J, Williams NS, et al. 2009. Small moleculemediated disruption of Wnt-dependent signaling in tissue regeneration and cancer. Nat Chem Biol 5: 100-107.

Ching W, Hang HC, Nusse R. 2008. Lipid-independent secretion of a Drosophila Wnt protein. J Biol Chem 283: 17092-17098.

Coombs GS, Yu J, Canning CA, Veltri CA, Covey TM, Cheong JK, Utomo V, Banerjee N, Zhang ZH, Jadulco RC, et al. 2010. WLS-dependent secretion of WNT3A requires Ser209 acylation and vacuolar acidification. $J$ Cell Sci 123: 3357-3367.

Coudreuse DY, Roel G, Betist MC, Destree O, Korswagen HC. 2006. Wnt gradient formation requires retromer function in Wnt-producing cells. Science 312: 921-924.

* Cruciat C-M, Niehrs C. 2012. Secreted and transmembrane Wnt inhibitors and activators. Cold Spring Harb Perspect Biol doi: 10.1101/cshperspect.a015081.

de Lau W, Barker N, Low TY, Koo BK, Li VS, Teunissen H, Kujala P, Haegebarth A, Peters PJ, van de Wetering M, et al. 2011. Lgr5 homologues associate with Wnt receptors and mediate R-spondin signalling. Nature 476: 293-297.

Fear MW, Kelsell DP, Spurr NK, Barnes MR. 2000. Wnt-16a, a novel Wnt-16 isoform, which shows differential expression in adult human tissues. Biochem Biophys Res Commun 278: 814-820.

Franch-Marro X, Wendler F, Griffith J, Maurice MM, Vincent JP. 2008a. In vivo role of lipid adducts on Wingless. $J$ Cell Sci 121: 1587-1592.

Franch-Marro X, Wendler F, Guidato S, Griffith J, BaenaLopez A, Itasaki N, Maurice MM, Vincent JP. 2008b. Wingless secretion requires endosome-to-Golgi retrieval of Wntless/Evi/Sprinter by the retromer complex. Nat Cell Biol 10: 170-177.

Fu J, Jiang M, Mirando AJ, Yu HM, Hsu W. 2009. Reciprocal regulation of Wnt and Gpr177/mouse Wntless is required for embryonic axis formation. Proc Natl Acad Sci 106: $18598-18603$.

Fuerer C, Habib SJ, Nusse R. 2010. A study on the interactions between heparan sulfate proteoglycans and Wnt proteins. Dev Dyn 239: 184-190.

Gasnereau I, Herr P, Chia PZ, Basler K, Gleeson PA. 2011. Identification of an endocytosis motif in an intracellular loop of Wntless, essential for its recycling and the control of Wnt signalling. J Biol Chem 286: 43324-43333.

Glinka A, Dolde C, Kirsch N, Huang YL, Kazanskaya O, Ingelfinger D, Boutros M, Cruciat CM, Niehrs C. 2011. LGR4 and LGR5 are R-spondin receptors mediating Wnt/ $\beta$-catenin and Wnt/PCP signalling. EMBO Rep 12: $1055-1061$.

Goodman RM, Thombre S, Firtina Z, Gray D, Betts D, Roebuck J, Spana EP, Selva EM. 2006. Sprinter: A novel transmembrane protein required for $\mathrm{Wg}$ secretion and signaling. Development 133: 4901-4911.

Grzeschik KH, Bornholdt D, Oeffner F, Konig A, del Carmen Boente M, Enders H, Fritz B, Hertl M, Grasshoff U, Hofling K, et al. 2007. Deficiency of PORCN, a regulator of Wnt signaling, is associated with focal dermal hypoplasia. Nat Genet 39: 833-835. 
Hacker U, Lin X, Perrimon N. 1997. The Drosophila sugarless gene modulates Wingless signaling and encodes an enzyme involved in polysaccharide biosynthesis. Development 124: 3565-3573.

Haerry TE, Heslip TR, Marsh JL, O'Connor MB. 1997. Defects in glucuronate biosynthesis disrupt Wingless signaling in Drosophila. Development 124: 3055-3064.

Han C, Belenkaya TY, Khodoun M, Tauchi M, Lin X. 2004 Distinct and collaborative roles of Drosophila EXT family proteins in morphogen signalling and gradient formation. Development 131: 1563-1575.

Harterink M, Port F, Lorenowicz MJ, McGough IJ, Silhankova M, Betist MC, van Weering JR, van Heesbeen RG, Middelkoop TC, Basler K, et al. 2011. A SNX3-dependent retromer pathway mediates retrograde transport of the Wnt sorting receptor Wntless and is required for Wnt secretion. Nat Cell Biol 13: 914-923.

He X, Saint-Jeannet JP, Wang Y, Nathans J, Dawid I, Varmus H. 1997. A member of the Frizzled protein family mediating axis induction by Wnt-5A. Science 275: 1652-1654

Herr P, Basler K. 2011. Porcupine-mediated lipidation is required for Wnt recognition by Wls. Dev Biol 361: $392-402$.

Hofmann K. 2000. A superfamily of membrane-bound $O$ acyltransferases with implications for Wnt signaling. Trends Biochem Sci 25: 111-112.

Itasaki N, Jones CM, Mercurio S, Rowe A, Domingos PM, Smith JC, Krumlauf R. 2003. Wise, a context-dependent activator and inhibitor of Wnt signalling. Development 130: 4295-4305.

Janda CY, Waghray D, Levin AM, Thomas C, Garcia KC. 2012. Structural basis of Wnt recognition by Frizzled. Science doi: 10.1126/science.1222879.

Junge HJ, Yang S, Burton JB, Paes K, Shu X, French DM, Costa M, Rice DS, Ye W. 2009. TSPAN12 regulates retinal vascular development by promoting Norrin- but not Wnt-induced FZD4/ $\beta$-catenin signaling. Cell 139: 299 311.

Kadowaki T, Wilder E, Klingensmith J, Zachary K, Perrimon N. 1996. The segment polarity gene porcupine encodes a putative multitransmembrane protein involved in Wingless processing. Genes Dev 10: 3116-3128.

Kang DW, Min do S. 2010. Positive feedback regulation between phospholipase $\mathrm{D}$ and Wnt signaling promotes Wnt-driven anchorage-independent growth of colorectal cancer cells. PLoS ONE 5: e12109.

Kang DW, Lee SH, Yoon JW, Park WS, Choi KY, Min do S 2010. Phospholipase D1 drives a positive feedback loop to reinforce the Wnt $/ \beta$-catenin/TCF signaling axis. Cancer Res 70: 4233-4242.

Kazanskaya O, Glinka A, del Barco Barrantes I, Stannek P, Niehrs C, Wu W. 2004. R-Spondin2 is a secreted activator of Wnt $/ \beta$-catenin signaling and is required for Xenopus myogenesis. Dev Cell 7: 525-534.

Kim KA, Zhao J, Andarmani S, Kakitani M, Oshima T, Binnerts ME, Abo A, Tomizuka K, Funk WD. 2006. RSpondin proteins: A novel link to $\beta$-catenin activation. Cell Cycle 5: 23-26.

Klein PS, Melton DA. 1996. A molecular mechanism for the effect of lithium on development. Proc Natl Acad Sci 93: $8455-8459$.
Komekado H, Yamamoto H, Chiba T, Kikuchi A. 2007. Glycosylation and palmitoylation of Wnt-3a are coupled to produce an active form of Wnt-3a. Genes Cells 12: $521-534$.

Korkut C, Ataman B, Ramachandran P, Ashley J, Barria R, Gherbesi N, Budnik V. 2009. Trans-synaptic transmission of vesicular Wnt signals through Evi/Wntless. Cell 139: 393-404.

Kurayoshi M, Yamamoto H, Izumi S, Kikuchi A. 2007. Posttranslational palmitoylation and glycosylation of Wnt-5a are necessary for its signalling. Biochem J 402: 515-523.

Liepinsh E, Banyai L, Patthy L, Otting G. 2006. NMR structure of the WIF domain of the human Wnt-inhibitory factor-1. J Mol Biol 357: 942-950.

Lin X, Perrimon N. 1999. Dally cooperates with Drosophila Frizzled 2 to transduce Wingless signalling. Nature 400: 281-284.

Liu H, Fergusson MM, Castilho RM, Liu J, Cao L, Chen J, Malide D, Rovira II, Schimel D, Kuo CJ, et al. 2007. Augmented Wnt signaling in a mammalian model of accelerated aging. Science 317: 803-806.

Malinauskas T. 2008. Docking of fatty acids into the WIF domain of the human Wnt inhibitory factor-1. Lipids 43: 227-230.

Mason JO, Kitajewski J, Varmus HE. 1992. Mutational analysis of mouse Wnt-1 identifies two temperature-sensitive alleles and attributes of Wnt-1 protein essential for transformation of a mammary cell line. Mol Biol Cell 3: 521-533.

Mathew D, Ataman B, Chen J, Zhang Y, Cumberledge S, Budnik V. 2005. Wingless signaling at synapses is through cleavage and nuclear import of receptor DFrizzled2. Science 310: 1344-1347.

Mikels AJ, Nusse R. 2006. Purified Wnt5a protein activates or inhibits $\beta$-catenin-TCF signaling depending on receptor context. PLoS Biol 4: e115.

Mulligan K, Fuerer C, Ching W, Fish M, Willert K, Nusse R. 2012. The Wnt/Wingless binding protein Swim promotes long-range signaling by maintaining Wingless solubility. Proc Natl Acad Sci 109: 370-377.

Nam JS, Turcotte TJ, Smith PF, Choi S, Yoon JK. 2006. Mouse cristin/R-spondin family proteins are novel ligands for the Frizzled 8 and LRP6 receptors and activate $\beta$-catenin-dependent gene expression. J Biol Chem 281: 13247-13257.

Neumann S, Coudreuse DY, van der Westhuyzen DR, Eckhardt ER, Korswagen HC, Schmitz G, Sprong H. 2009. Mammalian Wnt3a is released on lipoprotein particles. Traffic 10: $334-343$.

Nusse R, Varmus HE. 1982. Many tumors induced by the mouse mammary tumor virus contain a provirus integrated in the same region of the host genome. Cell 31: 99-109.

Nusse R, Brown A, Papkoff J, Scambler P, Shackleford G, McMahon A, Moon R, Varmus H. 1991. A new nomenclature for int-1 and related genes: The Wnt gene family. Cell 64: 231.

Panakova D, Sprong H, Marois E, Thiele C, Eaton S. 2005. Lipoprotein particles are required for Hedgehog and Wingless signalling. Nature 435: 58-65. 
Papkoff J, Brown AM, Varmus HE. 1987. The int-1 protooncogene products are glycoproteins that appear to enter the secretory pathway. Mol Cell Biol 7: 3978-3984.

Port F, Kuster M, Herr P, Furger E, Banziger C, Hausmann G, Basler K. 2008. Wingless secretion promotes and requires retromer-dependent cycling of Wntless. Nat Cell Biol 10: $178-185$.

Port F, Hausmann G, Basler K. 2011. A genome-wide RNA interference screen uncovers two $\mathrm{p} 24$ proteins as regulators of Wingless secretion. EMBO Rep 12: 1144-1152.

Prasad BC, Clark SG. 2006. Wnt signaling establishes anteroposterior neuronal polarity and requires retromer in C. elegans. Development 133: 1757-1766.

Sato N, Meijer L, Skaltsounis L, Greengard P, Brivanlou AH. 2004. Maintenance of pluripotency in human and mouse embryonic stem cells through activation of Wnt signaling by a pharmacological GSK-3-specific inhibitor. Nat Med 10: $55-63$.

Sepich DS, Usmani M, Pawlicki S, Solnica-Krezel L. 2011. Wnt/PCP signaling controls intracellular position of MTOCs during gastrulation convergence and extension movements. Development 138: 543-552.

Shibamoto S, Higano K, Takada R, Ito F, Takeichi M, Takada S. 1998. Cytoskeletal reorganization by soluble Wnt-3a protein signalling. Genes Cells 3: 659-670.

Silhankova M, Port F, Harterink M, Basler K, Korswagen HC. 2010. Wnt signalling requires MTM-6 and MTM9 myotubularin lipid-phosphatase function in Wnt-producing cells. EMBO J 29: 4094-4105.

Takada R, Satomi Y, Kurata T, Ueno N, Norioka S, Kondoh H, Takao T, Takada S. 2006. Monounsaturated fatty acid modification of Wnt protein: Its role in Wnt secretion. Dev Cell 11: 791-801.

Takei Y, Ozawa Y, Sato M, Watanabe A, Tabata T. 2004. Three Drosophila EXT genes shape morphogen gradients through synthesis of heparan sulfate proteoglycans. Development 131: 73-82.

Tao Q, Yokota C, Puck H, Kofron M, Birsoy B, Yan D, Asashima M, Wylie CC, Lin X, Heasman J. 2005. Maternal wnt1 1 activates the canonical Wnt signaling pathway required for axis formation in Xenopus embryos. Cell 120: 857-871.

Ten Berge D, Kurek D, Blauwkamp T, Koole W, Maas A, Eroglu E, Siu RK, Nusse R. 2011. Embryonic stem cells require Wnt proteins to prevent differentiation to epiblast stem cells. Nat Cell Biol 13: 1070-1075.

Topczewski J, Sepich DS, Myers DC, Walker C, Amores A, Lele Z, Hammerschmidt M, Postlethwait J, Solnica-Krezel L. 2001. The zebrafish glypican knypek controls cell polarity during gastrulation movements of convergent extension. Dev Cell 1: 251-264. van den Heuvel M, Nusse R, Johnston P, Lawrence PA. 1989. Distribution of the wingless gene product in Drosophila embryos: A protein involved in cell-cell communication. Cell 59: 739-749.

van den Heuvel M, Harryman-Samos C, Klingensmith J, Perrimon N, Nusse R. 1993. Mutations in the segment polarity genes wingless and porcupine impair secretion of the Wingless protein. EMBO J 12: 5293-5302.

van Leeuwen F, Samos CH, Nusse R. 1994. Biological activity of soluble wingless protein in cultured Drosophila imaginal disc cells. Nature 368: 342-344.

Wang X, Reid Sutton V, Omar Peraza-Llanes J, Yu Z, Rosetta R, Kou YC, Eble TN, Patel A, Thaller C, Fang P, et al. 2007. Mutations in X-linked PORCN, a putative regulator of Wnt signaling, cause focal dermal hypoplasia. Nat Genet 39: $836-838$.

Willert K, Brown JD, Danenberg E, Duncan AW, Weissman IL, Reya T, Yates JR 3rd, Nusse R. 2003. Wnt proteins are lipid-modified and can act as stem cell growth factors. Nature 423: 448-452.

Xu Q, Wang Y, Dabdoub A, Smallwood PM, Williams J, Woods C, Kelley MW, Jiang L, Tasman W, Zhang K, et al. 2004. Vascular development in the retina and inner ear: Control by Norrin and Frizzled-4, a high-affinity ligand-receptor pair. Cell 116: 883-895.

Yang PT, Lorenowicz MJ, Silhankova M, Coudreuse DY, Betist MC, Korswagen HC. 2008. Wnt signaling requires retromer-dependent recycling of MIG-14/Wntless in Wnt-producing cells. Dev Cell 14: 140-147.

Zecca M, Basler K, Struhl G. 1996. Direct and long-range action of a Wingless morphogen gradient. Cell 87: 833-844.

Zhang P, Wu Y, Belenkaya TY, Lin X. 2011. SNX3 controls Wingless/Wnt secretion through regulating retromerdependent recycling of Wntless. Cell Res 21: 1677-1690.

Zhang X, Abreu JG, Yokota C, MacDonald BT, Singh S, Coburn KL, Cheong S-M, Zhang MM, Ye Q-Z, Hang $\mathrm{HC}$, et al. 2012. Tikil is required for head formation via Wnt cleavage-oxidation and inactivation. Cell 149: 1565-1577.

Ziegler S, Rohrs S, Tickenbrock L, Moroy T, Klein-Hitpass L, Vetter IR, Muller O. 2005. Novel target genes of the Wnt pathway and statistical insights into Wnt target promoter regulation. FEBS J 272: 1600-1615.

Ziegler S, Rohrs S, Tickenbrock L, Langerak A, Chu ST, Feldmann I, Jakubowski N, Muller O. 2007. Lipocalin $24 \mathrm{p} 3$ is regulated by the Wnt pathway independent of regulation by iron. Cancer Genet Cytogenet 174: 16-23.

Zoltewicz JS, Ashique AM, Choe Y, Lee G, Taylor S, Phamluong K, Solloway M, Peterson AS. 2009. Wnt signaling is regulated by endoplasmic reticulum retention. PLoS ONE 4: e6191. 


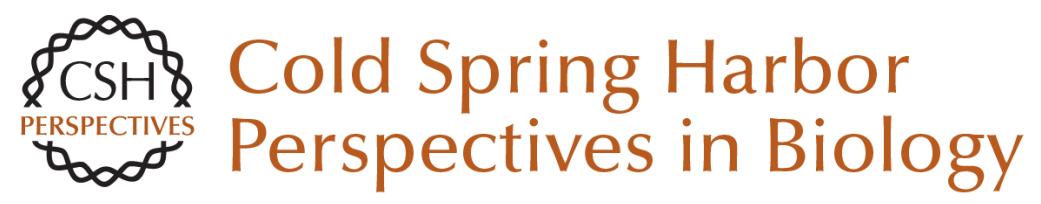

\section{Wnt Proteins}

Karl Willert and Roel Nusse

Cold Spring Harb Perspect Biol 2012; doi: 10.1101/cshperspect.a007864

For additional articles in this collection, see http://cshperspectives.cshlp.org/cgi/collection/

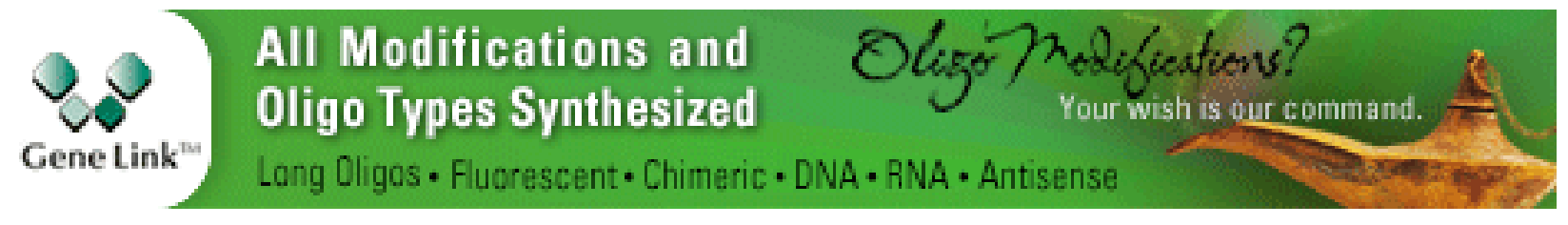

Copyright @ 2012 Cold Spring Harbor Laboratory Press; all rights reserved 